\title{
The History of Solar Prominence Research: Historical Introduction to IAU Colloquium No. 167 "New Perspectives on Solar Prominences"
}

\author{
Einar Tandberg-Hanssen \\ NASA Marshall Space Flight Center, Huntsville, AL 35812, USA
}

\begin{abstract}
We trace the history of observational solar prominence research from the early period when total solar eclipses provided the only means of observation, via the introduction of photography and spectroscopy in the 1860's, to the present epoch when application of the Zeeman and the Hanle effects makes possible determination of the allimportant magnetic fields in prominences.

Dividing prominence models into two classes, those that treat the support of the prominence against gravity and those concerned with the physical conditions in the prominence plasma itself, we describe the progress made in both classes of models since the 1950's, when the first models were proposed. Finally, we comment on the necessity of combining aspects of the two classes of prominence models into a comprehensive picture that can account for the global nature of prominence support and stability, and where the adjacent coronal structures play an important role.
\end{abstract}

\section{Introduction}

About 150 years ago astronomers, for the first time, provided the correct albeit incomplete - interpretation of the prominence phenomenon as "clouds in the solar corona." However, prominences had been observed since the Middle Ages, and explanations had at times been exotic.

At this colloquium, we are concentrating on those objects that fall into the category of quiescent prominences, i.e., the large, rather stable structures that, when observed on the solar disk in absorption (e.g., $\mathrm{H} \alpha$ ), are often referred to as filaments.

Figure 1 may be taken as a defining picture of a quiescent prominence. Taken near solar activity minimum, it shows the prominence as part of the polar crown. Closer to activity maximum one also sees many smaller active-region filaments as shown in Figure 2, which also displays a quiescent prominence seen abovethe southeastern limb. These are the objects we shall keep in mind during the discussions at this colloquium.

For convenience, I shall first discuss the history of prominence research from an observational point of view by dividing the progress, somewhat arbitrarily, into three time periods, namely: 


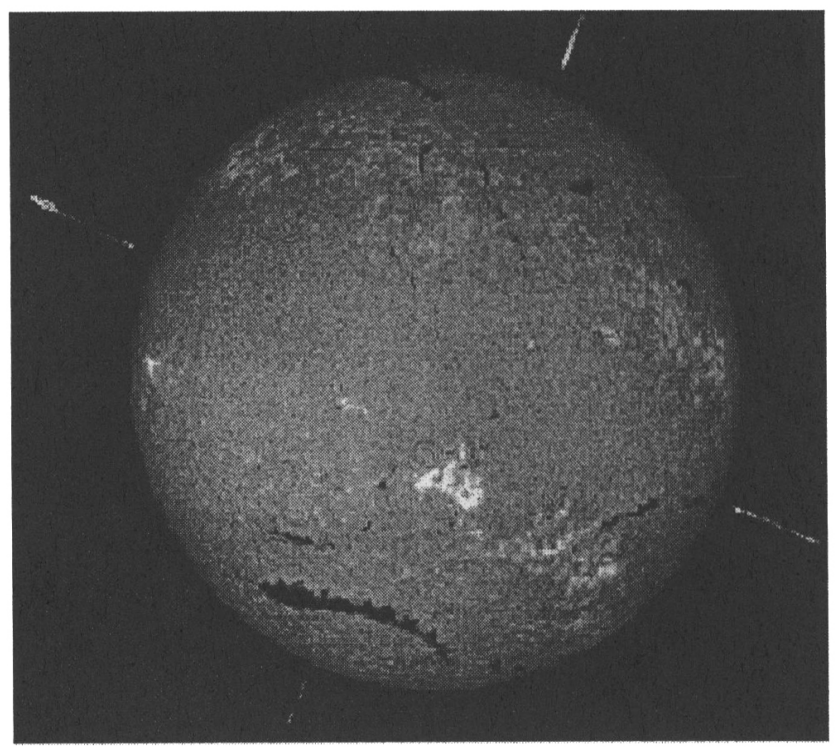

Figure 1. Large quiescent prominence, part of the (south) polar crown, near solar minimum (courtesy Observatoire de Paris-Meudon and P. Mein).

(1) The speculative period, roughly before 1860 ;

(2) The spectrographic period, roughly between 1860 and 1960 ; and

(3) The polarimetric period, roughly after 1960.

Table 1. Historic Eclipse Observations

\begin{tabular}{lll} 
Year (site) & Observer & Interpretation \\
\hline 1239 & Muratori & "burning hole in the corona" \\
1733 (Sweden) & Vassenius & "clouds in lunar atmosphere" \\
1788 & Ulloa & "hole in the Moon" \\
1842 (France, Italy) & Arago, Struve, etc. & "prominences a solar phenomenon" \\
1851 (Norway, Sweden) & Airy, Carrington & "solar cloud formation" \\
\hline 1860 (Spain) & Photography introduced & \\
1868 (India, Malacca) & Spectroscopy introduced &
\end{tabular}

\section{The Speculative Period}

The only way prominences could be observed during this period was during total solar eclipses. In 1239 Muratori (see Secchi 1875) observed the corona during an eclipse and reported a burning hole. This "burning hole" in all probability was a prominence, or a cavity with a prominence inside it.

Medieval Russian chronicles (see Vyssotsky 1949) also mention prominen- 


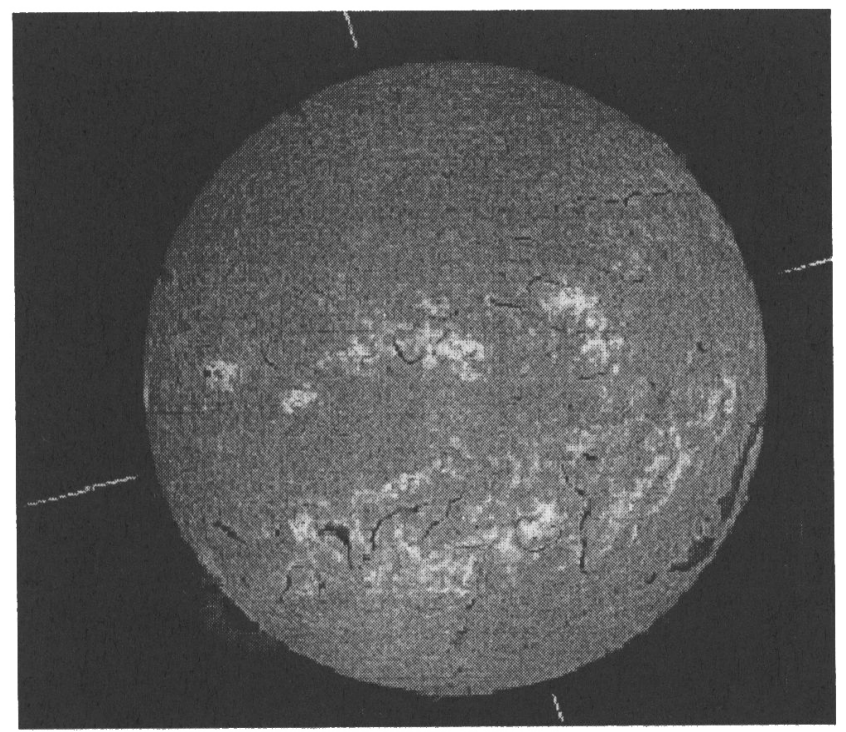

Figure 2. Active and quiescent prominences, near solar maximum (courtesy Observatoire de Paris-Meudon and P. Mein).

ces, but the first semi-scientific description came after the eclipse of May 2, 1733 in Sweden. During this event, Vassenius (1733) observed three or four prominences and described them as reddish clouds in the lunar atmosphere. Ulloa (1779) observed what probably was an active prominence during the eclipse of 1778 and attributed it to a hole in the Moon.

These early observations of prominences were subsequently all but forgotten, and all the experienced astronomers were taken by surprise when they rediscovered the phenomenon during the eclipse of July 8, 1842 in France and Italy. Even though they realized that this must be a solar phenomenon, their descriptions of the shape of prominences were so vague that they could not prevent others from believing that prominences were mountains on the Sun (see Grant 1852).

Not until the eclipse in 1851, observed in Norway and Sweden, did a correct, if incomplete, interpretation emerge: prominences are solar cloud formations. Thus, in 4 years (2001) we can celebrate the sesquicentennial anniversary of a correct interpretation, even though the detailed physical nature of these clouds is still being debated, which is the reason we are all here in Aussois today.

\section{The Spectrographic Period}

Spectroscopy was first used in eclipse observation in 1868 , but I have let this period start in 1860 when photography was introduced during the eclipse in Spain, since the former technique depended on the latter. In both cases the recogni- 
tion goes to Secchi (1868) and de la Rue (1868), who employed spectrographic methods on August 18, 1868 at the eclipse in India and Malacca to study the "chemistry of prominences." It was found that the spectrum of prominences consisted of bright lines, and from then on prominences were considered to be glowing masses of gas.

An interesting development followed immediately after the eclipse. Janssen (1868) realized that many of the emission lines were so brilliant that they should be visible without an eclipse. The next day he placed the slit of the spectrograph outside the limb of the Sun's image and observed prominence emission lines in full daylight. Independently and simultaneously, the same kind of observation was reported by Lockyer (1868a, b), who with Huggins, had tried to accomplish this for some time.

The 1868 eclipse also brought the discovery of the "open-slit" method, giving a series of monochromatic images of a prominence, corresponding to the emission lines observed with a normal slit. As a result of all these developments, the 1870's saw a great increase in knowledge of prominences, as also witnessed by Secchi's seminal book, Le Soleil (Secchi 1875-77).

Then in 1892, Hale invented the spectroheliograph (Hale and Ellerman 1903) and the following year Deslandres had his first instrument ready in France (Deslandres 1910). The importance of this kind of instrument is beautifully demonstrated in Figures 1 and 2 in this article.

In 1899 Deslandres hired 16-year-old Lucien d'Azambuja who soon became very adept, both as a mechanic and later as a bona fide scientist, and followed in Deslandres' footsteps.

The next great developments were due to Bernard Lyot who, with the invention of the birefringent filter and the coronagraph (Lyot 1936), made possible modern-day observations of prominences at any time without an eclipse. Figure 3 reproduces some of his early observations (June 1937) that also made possible the study of prominence development. The vast amount of data on prominences was brilliantly treated in the 1948 paper by the d'Azambujas: Etude d'Ensemble des Protubérances Solaires et de leur Evolution, which remains a standard reference (d'Azambuja and d'Azambuja 1948). It includes the disparition brusque phenomenon, which probably was first photographed by Deslandres in 1897 (Deslandres 1897) and studied by him with a spectroheliograph in 1899, and which will be lively debated at this colloquium.

\section{Prominence Drawings}

Before we embark on a discussion of the polarimetric period, a word on prominence drawings may be in order. Early drawings were often quite fanciful. Figure 4 shows a 17 th century masterpiece from Kircher's "Mundus Subterraneus" of 1665 .

With the introduction of photography in 1860 , one might suppose that drawings of prominences seen during a total eclipse would lose their raison d'tre. However, for many years that was not the case. The introduction of photography was certainly a great step forward, but early photographs often could not compete with visual inspection of prominence fine-structure. Secchi himself was a master, and Figure 5 reproduces one of his well-known drawings. 


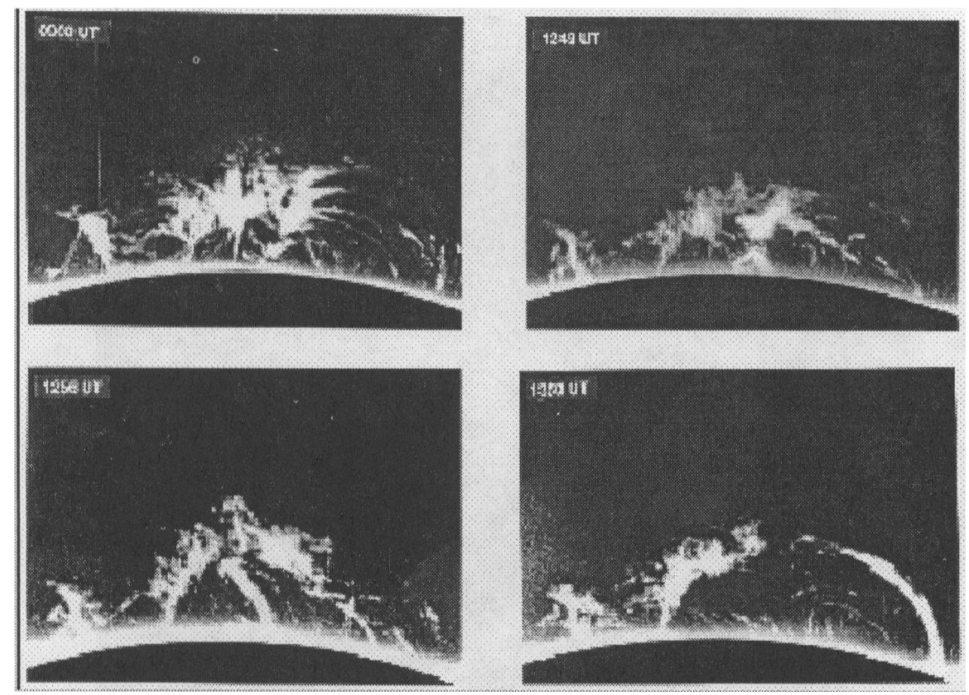

Figure 3. Prominence photographs obtained by Lyot in 1937 with his coronagraph (courtesy Observatoire de Paris).

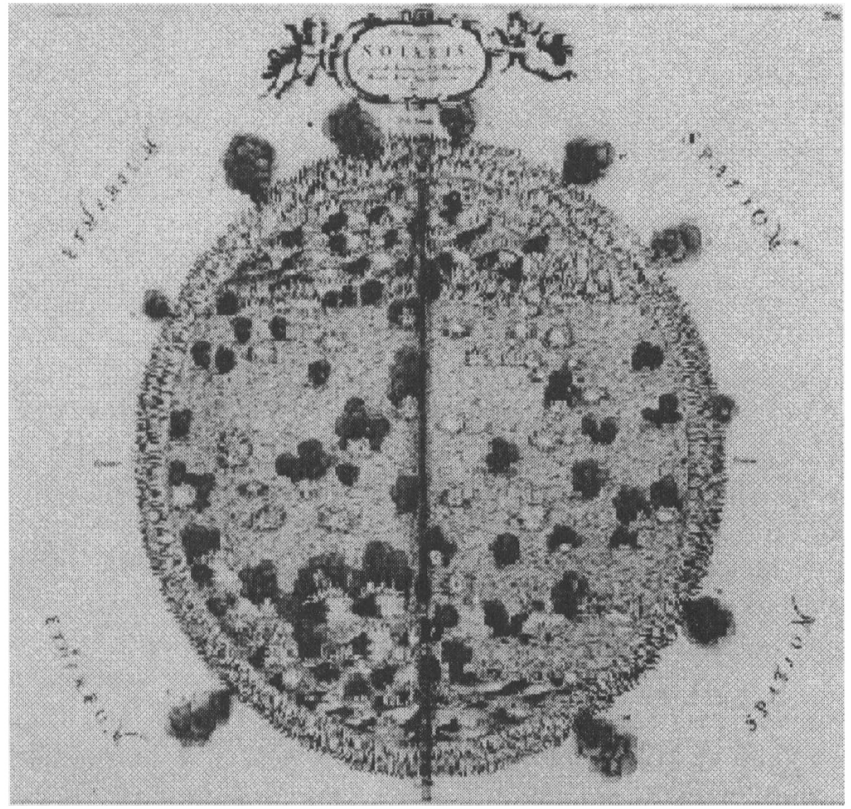

Figure 4. 17th century drawing from Kircher's Mundus Subterraneus, 1665 (courtesy Observatoire de Paris). 


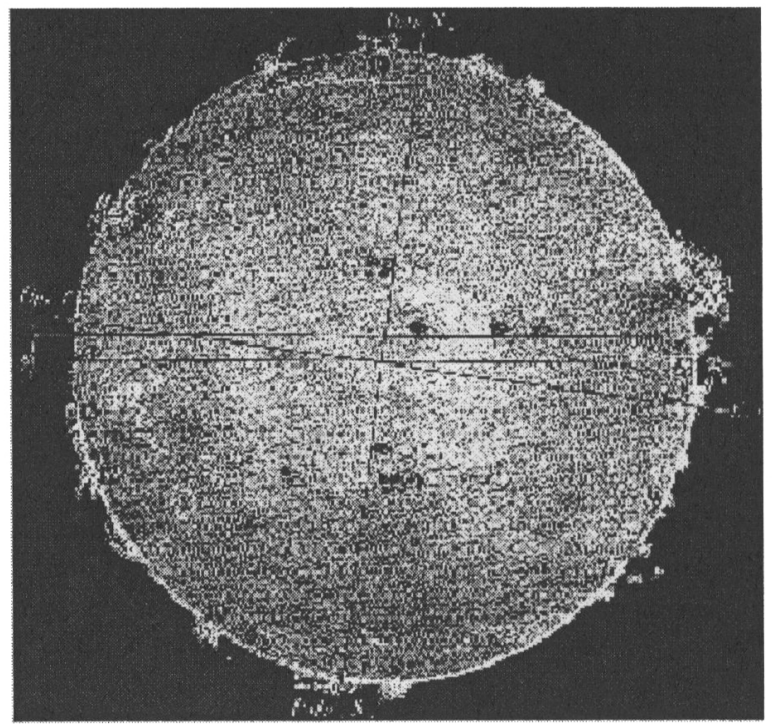

Figure 5. Drawing of prominences observed at total solar eclipse (Secchi in Le Soleil, 1877).

Figure 6 reproduces some other early drawings made by Fearnley in Norway in 1872-73, showing a disparition brusque, a quarter of a century before Deslandres' photographic observations.

\section{The Polarimetric Period}

This period started in 1961 when Zirin and Severny made the first measurements of magnetic fields in prominences, using the Zeeman effect. Already in 1908, Hale had recorded the magnetic field in sunspots from Zeeman line displacements; later polarization characteristics of the Zeeman effect made it possible to observe the much weaker prominence fields, which is the basis for one of the two methods still being used.

In the 1970's, extensive Zeeman-effect measurements of prominences were performed at the High Altitude Observatory's Climax station, while great improvements were made by the French groups at Meudon and Pic-du-Midi, using the Hanle effect, the other method in current use. The magnetic fields in prominences were found to range from a few gauss to more than 100 gauss. Furthermore, it became clear that the field in quiescent prominences has an important component along the long axis of the prominence. We are still reaping the rich harvest of these efforts as the polarimetric period continues. 


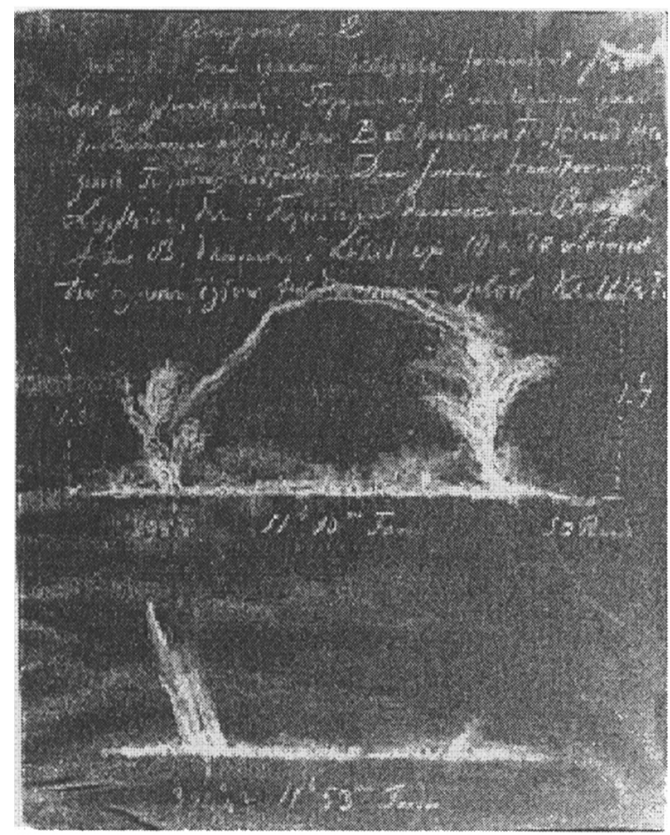

Figure 6. Prominence drawing made by Fearnley in Norway, 1872-73 (courtesy Inst. for Theoretical Astrophysics, Oslo and O. Engvold).

\section{Development of Prominence Models, Interpretations, and Theory}

During the later part of the spectroscopic period, serious discussions took place as to the nature of prominences, and models were constructed to explain their characteristics. Prominence models may be divided into two classes: those that are concerned with the support of the prominence in the solar atmosphere, and those that describe the internal, thermodynamic structure of the prominence plasma.

\subsection{Prominence Support by Magnetic Fields}

In 1951 - 100 years after the first, roughly correct, interpretation of the prominence phenomenon - Menzel presented his model, showing that the prominence could be supported in an assumed magnetic field; i.e., due to an equilibrium between the Lorentz force and gravity (Bhatnagar et al. 1951). Refinements followed quickly: Dungey's model, 1953 (Dungey 1953) and then the now-famous Kippenhahn-Schlüter model in 1957 (Kippenhahn and Schlüter 1957). In 1972, Anzer (1972) opened a new phase in the modeling research by using observed magnetic fields and developing a method to calculate the electric current in quiescent prominences. Finally, as far as this overview is concerned, in 1974 came the Kuperus and Raadu model for the so-called inverse-polarity prominences (Kuperus and Raadu 1974). Notice that all these models are two-dimensional. 


\subsection{Internal Structure}

More or less hand-in-hand with these developments in support mechanisms went research into the nature of the prominence plasma that was being supported. The early models of the plasma ignored the magnetic field but analyzed the temperature, density, and velocity structures; namely, the models due to de Jager (1959), Ivanov-Kholodny (1959), Jefferies and Orrall (1963), and Hirayama (1963). By the mid 1960's we could fairly well describe the thermodynamic nature of prominence plasmas.

\subsection{Towards a Comprehensive Prominence Model}

The last 20 years have seen increasingly sophisticated attempts at combining studies of the internal structures with analyses of magnetic-field supporting mechanisms. It is not the place in this historical introduction to go into details concerning these comprehensive model attempts. We shall hear about them in the days ahead, since the authors of several of these beautiful investigations are present here in Aussois. Of prime importance in these studies is the existence of a longitudinal component, and three-dimensional aspects, of the magnetic field in the prominence; a situation that entails the creation of helical fields, and points to magnetic reconnection as a basic mechanism in prominence behavior, intimately linked to the large-scale magnetic structure in the corona above and around the prominence (i.e., the helmet streamer with its coronal cavity). We shall surely hear more about these aspects as this colloquium proceeds.

Acknowledgments. I thank R. Moore for reading the manuscript and suggesting several improvements.

\section{References}

Anzer, U. 1972, Solar Phys., 24, 324

d'Azambuja, L. and d'Azambuja, M. 1948, Ann. Obs. Paris-Meudon, 6, 7

Bhatnagar, P. L., Krook, M., and Menzel, D. H. 1951, Dynamics of Ionized Media, Rep. Conf. Univ. College, London

de Jager, C. 1959, Handbuch der Physik, 52, 80

de la Rue, W. 1868, MNRAS, 29, 73

Deslandres, H. 1897, Compt. Rend. Acad. Sci., 124, 171

Deslandres, H. 1910, Ann. Obs. Paris-Meudon, 4, I, 1

Dungey, J. W. 1953, MNRAS, 113, 180

Grant, R. 1852, History of Physical Astronomy, Robert Baldwin: London

Hale, G. E., and Ellerman, F. 1903, Publ. Yerkes Obs., 3, Part 1, 3

Hirayama, T. 1963, PASJ, 15, 122

Ivanov-Kholodny, G. S. 1959, AZh, 36, 589

Janssen, P. J. 1868, Compt. Rend. Acad. Sci., 67, 838

Jefferies, J. T. and Orrall, F. Q. 1963, ApJ, 137, 1232

Kippenhahn, R. and Schlüter, A. 1957, ZAp, 43, 36

Kuperus, M. and Raadu, M. A. 1974, A\&A, 31, 189 
Lockyer, J. N. 1868a, Compt. Rend. Acad. Sci., 67, 836

Lockyer, J. N. 1868b, Compt. Rend. Acad. Sci., 67, 949

Lyot, B. 1936, Compt. Rend. Acad. Sci., 202, 392.

Secchi, A. 1868, Compt. Rend. Acad. Sci. 66, 396.

Secchi, A. 1875-77, Le Soleil, Gauthier-Villars, Paris, Vols. 1 and 2

Ulloa, A. 1779, Phil. Trans. Roy. Soc. London, 69, 105

Vassenius, B. 1733, Phil. Trans. Roy. Soc. London, 38, 134

Vyssotsky, A. N. 1949, Meddelanden Lund. Obs., 2(126), 9 